VoL. 59 (1999) [315-322]

\title{
A GENERALISATION OF THE SUMMATION FORMULA OF PLANA
}

\section{ClÉment FrapPier}

An extension of the classical summation formula of Plana is obtained. The extension is obtained by using the zeros of a Bessel function of the first kind.

\section{INTRODUCTION}

Let $f(z)$ be analytic in the half-plane $\operatorname{Re}(z) \geqslant 0$ and let either the series $\sum_{k=0}^{\infty} f(k)$ or the integral $\int_{0}^{\omega} f(x) d x$ be convergent. Suppose, in addition, that

(A) $\lim _{N \rightarrow \infty}|f(x \pm i N)| e^{-2 \pi N}=0$ uniformly in $x$ on every finite interval, and

(B) $\int_{0}^{\infty}|f(M \pm i y)| e^{-2 \pi y} d y$ exists for every $M \geqslant 0$ and tends to zero as $M \rightarrow \infty$.

Under these assumptions, we have

$$
\sum_{k=0}^{\infty} f(k)=\frac{1}{2} f(0)+\int_{0}^{\infty} f(x) d x+i \int_{0}^{\infty} \frac{f(i y)-f(-i y)}{e^{2 \pi y}-1} d y .
$$

Equation (1) is known as the summation formula of Plana. It has applications to the $\Gamma$ function, the Riemann $\zeta$ function and the discrete Laplace transform [4].

The aim of this paper is to present a generalisation of (1). Our results involve a parameter $\alpha>-1$, the order of a Bessel function of the first kind. We obtain (1) as the special case $\alpha=1 / 2$. For $\alpha=-1 / 2$ we shall obtain a formula similar to (1), namely

$$
\sum_{k=1}^{\infty} f\left(k-\frac{1}{2}\right)=\int_{0}^{\infty} f(x) d x-i \int_{0}^{\infty} \frac{f(i y)-f(-i y)}{e^{2 \pi y}+1} d y .
$$

We examine some examples, one of which leads us to consider, in a natural way, an extension of the Riemann $\zeta$ function. Some fragmentary properties of this function are given, including a functional equation.

\section{Received 28th September, 1998}

This research was supported by the Natural Sciences and Engineering Research Council of Canada Grant No. OGP0009331.

Copyright Clearance Centre, Inc. Serial-fee code: 0004-9729/99 $\$ A 2.00+0.00$. 


\section{The summation formula}

Before we state our results, it is convenient to recall some standard notation. Let $\alpha>-1$ be a real number. The function $J_{\alpha}$ defined by the relation

$$
\frac{J_{\alpha}(z)}{z^{\alpha}}=\sum_{k=0}^{\infty} \frac{(-1)^{k} z^{2 k}}{2^{2 k+\alpha} k ! \Gamma(\alpha+k+1)}
$$

is known as the Bessel function of the first kind, of order $\alpha$. The function $\left(J_{\alpha}(z) / z^{\alpha}\right)$ is an even entire function of exponential type 1, whose zeros $j_{k}=j_{k}(\alpha)$ are real and simple [5, Chapter XV]. We arrange these zeros such that $0<j_{1}<j_{2}<\ldots$ and $j_{-k}=-j_{k}$. The Bessel function of the second kind $Y_{\alpha}$ (Weber's function) is defined, whenever $\alpha$ is not an integer, by

$$
Y_{\alpha}(z)=\frac{\cos (\alpha \pi) J_{\alpha}(z)-J_{-\alpha}(z)}{\sin (\alpha \pi)} .
$$

This function is often denoted $N_{\alpha}$ (Neumann's function). The Bessel functions of the third kind $H_{\alpha}^{(1)}$ and $H_{\alpha}^{(2)}$ (Hankel's functions) are defined by

$$
H_{\alpha}^{(1)}(z)=J_{\alpha}(z)+i Y_{\alpha}(z)=i \frac{\left(e^{-\alpha \pi i} J_{\alpha}(z)-J_{-\alpha}(z)\right)}{\sin (\alpha \pi)}
$$

and

$$
H_{\alpha}^{(2)}(z)=J_{\alpha}(z)-i Y_{\alpha}(z)=i \frac{\left(J_{-\alpha}(z)-e^{\alpha \pi i} J_{\alpha}(z)\right)}{\sin (\alpha \pi)}
$$

Now let $f(z)$ be analytic for $\operatorname{Re}(z) \geqslant 0$. We define a real number $\omega=\omega(\alpha)$ by

$$
\omega= \begin{cases}2 \alpha-1 & \text { if } \quad \alpha>0 \\ -1 & \text { if } \quad \alpha<0\end{cases}
$$

Suppose that

(a) $\lim _{N \rightarrow \infty}(x \pm i N)^{\omega} f(x \pm i N) e^{-2 N}=0$ uniformly in $x$ on every finite interval, and

(b) $\int_{0}^{\infty}\left|(M \pm i y)^{\omega} f(M \pm i y)\right| e^{-2 y} d y$ exists for every $M \geqslant 0$ and tends to zero as $M \rightarrow \infty$.

ThEOREM 1. Let $\alpha>0$ be a real number, not an integer, and let $f(z)$ be analytic for $\operatorname{Re}(z) \geqslant 0$. Suppose that the series $\sum_{k=1}^{\infty}\left(j_{k}^{2 \alpha-2} f\left(j_{k}\right) /\left(J_{\alpha}^{\prime}\left(j_{k}\right)\right)^{2}\right)$ or the integral $\int_{0}^{\infty} x^{2 \alpha-1} f(x) d x$ is convergent. If conditions (a) and (b) are satisfied then

$$
\begin{aligned}
& 2 \sum_{k=1}^{\infty} \frac{j_{k}^{2 \alpha-2} f\left(j_{k}\right)}{\left(J_{\alpha}^{\prime}\left(j_{k}\right)\right)^{2}}=\int_{0}^{\infty} x^{2 \alpha-1} f(x) d x-2^{2 \alpha-1} \Gamma(\alpha+1) \Gamma(\alpha) f(0) \\
& \quad-\frac{i}{2 \sin (\alpha \pi)} \int_{0}^{\infty} y^{2 \alpha-1} \frac{\left(J_{\alpha}(i y)-e^{\alpha \pi i} J_{-\alpha}(i y)\right)}{J_{\alpha}(i y)}(f(i y)-f(-i y)) d y .
\end{aligned}
$$


In the special case $\alpha=1 / 2$ we have $J_{1 / 2}(z)=\sqrt{2 /(\pi z)} \sin (z), j_{k}=k \pi$, and (8), where $f(z)$ is replaced by $f(z / \pi)$, reduces to (1). For $\alpha=3 / 2$ we have $J_{3 / 2}(z)=$ $\sqrt{2 /(\pi z)}((\sin (z) / z)-\cos (z)), \tan \left(j_{k}\right)=j_{k}$, and (8) becomes

$$
\sum_{k=1}^{\infty}\left(1+j_{k}^{2}\right) f\left(j_{k}\right)=\int_{0}^{\infty} x^{2} f(x) d x-\frac{3 \pi}{2} f(0)+i \int_{0}^{\infty} \frac{y^{2}(y+1)(f(i y)-f(-i y))}{(y-1) e^{2 y}+(y+1)} d y
$$

ThEOREM 2. Let $-1<\alpha<0$ be a real number and let $f(z)$ be analytic for $R e(z) \geqslant 0$, with $f(0)=0$. Suppose that the series $\sum_{k=1}^{\infty}\left(f\left(j_{k}\right) / j_{k}^{2}\left(J_{\alpha}^{\prime}\left(j_{k}\right)\right)^{2}\right)$ or the integral $\int_{0}^{\infty}(f(x) / x) d x$ is convergent. If conditions (a) and (b) are satisfied then

$$
\begin{aligned}
2 \sum_{k=1}^{\infty} \frac{f\left(j_{k}\right)}{j_{k}^{2}\left(J_{\alpha}^{\prime}\left(j_{k}\right)\right)^{2}} & =\int_{0}^{\infty} \frac{f(x)}{x} d x \\
& +\frac{1}{2 \sin (\alpha \pi)} \int_{0}^{\infty} \frac{\left(J_{\alpha}(i y)-e^{\alpha \pi i} J_{-\alpha}(i y)\right)}{J_{\alpha}(i y)} \frac{\left(e^{-\alpha \pi i} f(i y)-e^{\alpha \pi i} f(-i y)\right)}{i y} d y .
\end{aligned}
$$

The particular case $\alpha=-1 / 2$ of $(10)$ gives, since $J_{-1 / 2}(z)=\sqrt{2 /(\pi z)} \cos (z)$ and $j_{k}=(2 k-1)(\pi / 2)$, the summation formula

$$
\sum_{k=1}^{\infty} \frac{f\left(\left(k-\frac{1}{2}\right) \pi\right)}{\left(k-\frac{1}{2}\right)}=\int_{0}^{\infty} \frac{f(x)}{x} d x-\int_{0}^{\infty} \frac{(f(i y)+f(-i y))}{y\left(e^{2 y}+1\right)} d y
$$

from which (2) follows if we replace $f(z)$ by $z f(z / \pi)$. Of course, a small modification to the hypothesis of Theorem 2 must then be made.

\section{PROOFS OF THE THEOREMS}

For the various properties of Bessel functions used in the proofs, we refer the reader to $[5]$.

Let $\varepsilon$ be a small positive number and let $M, N$ be large positive numbers with $M:=m \pi+\alpha(\pi / 2)+(\pi / 4)$ for some positive integer $m$. We consider, in the complex plane, the closed curve $\Gamma=\Gamma(M, N, \varepsilon)$ which is the union of the intervals $[M-i N, M+i N]$, $[M+i N, i N],[i N, i \varepsilon],[-i \varepsilon,-i N],[-i N, M-i N]$ and the semicircle $C(\varepsilon)=\{z:|z|=$ $\varepsilon, \operatorname{Re}(z) \geqslant 0\}$. Thus, $\Gamma$ is a rectangular curve, in the right half-plane, with corners $M \pm i N, \pm i N$, except that the interval $[i \varepsilon,-i \varepsilon]$ is replaced by $C(\varepsilon)$. The curve $\Gamma$ is oriented positively. Let also $\Gamma_{1}:=\{z \in \Gamma: \operatorname{Im}(z) \geqslant 0\}$ and $\Gamma_{2}:=\{z \in \Gamma: \operatorname{Im}(z) \leqslant 0\}$, so that $\Gamma=\Gamma_{1} \cup \Gamma_{2}$.

The function $z^{\omega} f(z)\left(Y_{\alpha}(z) / J_{\alpha}(z)\right)$ is analytic on and inside $\Gamma$, except for simple poles at $z=j_{k}, 1 \leqslant k \leqslant m$. By the residue theorem, we have

$$
\frac{1}{2 \pi i} \int_{\Gamma} z^{\omega} f(z) \frac{Y_{\alpha}(z)}{J_{\alpha}(z)} d z=-\frac{2}{\pi} \sum_{k=1}^{m} \frac{j_{k}^{\omega-1} f\left(j_{k}\right)}{\left(J_{\alpha}^{\prime}\left(j_{k}\right)\right)^{2}}
$$


The relation $Y_{\alpha}\left(j_{k}\right)=-J_{-\alpha}\left(j_{k}\right) / \sin (\alpha \pi)=-2 /\left(\pi j_{k} J_{\alpha}^{\prime}\left(j_{k}\right)\right)$ has been used to compute the residue at $z=j_{k}$. It follows from (12) that

$$
\begin{aligned}
-\frac{2}{\pi} \sum_{k=1}^{m} \frac{j_{k}^{\omega-1} f\left(j_{k}\right)}{\left(J_{\alpha}^{\prime}\left(j_{k}\right)\right)^{2}}= & \frac{1}{2 \pi i} \int_{\Gamma_{1}} z^{\omega} f(z) \frac{Y_{\alpha}(z)}{J_{\alpha}(z)} d z+\frac{1}{2 \pi i} \int_{\Gamma_{2}} z^{\omega} f(z) \frac{Y_{\alpha}(z)}{J_{\alpha}(z)} d z \\
= & -\frac{1}{2 \pi} \int_{\Gamma_{1}} z^{\omega} f(z) \frac{H_{\alpha}^{(1)}(z)}{J_{\alpha}(z)} d z+\frac{1}{2 \pi} \int_{\Gamma_{1}} z^{\omega} f(z) d z \\
& +\frac{1}{2 \pi} \int_{\Gamma_{2}} z^{\omega} f(z) \frac{H_{\alpha}^{(2)}(z)}{J_{\alpha}(z)} d z-\frac{1}{2 \pi} \int_{\Gamma_{2}} z^{\omega} f(z) d z .
\end{aligned}
$$

Cauchy's theorem, applied to the closed curves $\Gamma_{1} \cup[\varepsilon, M]$ and $\Gamma_{2} \cup[M, \varepsilon]$, gives

$$
\int_{\Gamma_{1}} z^{\omega} f(z) d z=-\int_{\varepsilon}^{M} x^{\omega} f(x) d x
$$

and

$$
\int_{\Gamma_{2}} z^{\omega} f(z) d z=\int_{\varepsilon}^{M} x^{\omega} f(x) d x
$$

Substituting in (13), we obtain

$$
\begin{aligned}
-\frac{2}{\pi} \sum_{k=1}^{m} \frac{j_{k}^{\omega-1} f\left(j_{k}\right)}{\left(J_{\alpha}^{\prime}\left(j_{k}\right)\right)^{2}}= & -\frac{1}{\pi} \int_{\varepsilon}^{M} x^{\omega} f(x) d x-\frac{1}{2 \pi} \int_{\Gamma_{1}} z^{\omega} f(z) \frac{H_{\alpha}^{(1)}(z)}{J_{\alpha}(z)} d z \\
& +\frac{1}{2 \pi} \int_{\Gamma_{2}} z^{\omega} f(z) \frac{H_{\alpha}^{(2)}(z)}{J_{\alpha}(z)} d z .
\end{aligned}
$$

In (16), the integral along $\Gamma_{1}$ may be written explicitly as

$$
\begin{aligned}
\int_{\Gamma_{1}} z^{\omega} f(z) \frac{H_{\alpha}^{(1)}(z)}{J_{\alpha}(z)} d z=i \int_{0}^{N}(M+i y)^{\omega} f(M+i y) \frac{H_{\alpha}^{(1)}(M+i y)}{J_{\alpha}(M+i y)} d y \\
+\int_{M}^{0}(x+i N)^{\omega} f(x+i N) \frac{H_{\alpha}^{(1)}(x+i N)}{J_{\alpha}(x+i N)} d x \\
+i \int_{N}^{\varepsilon}(i y)^{\omega} f(i y) \frac{H_{\alpha}^{(1)}(i y)}{J_{\alpha}(i y)} d y \\
+i \int_{\pi / 2}^{0}\left(\varepsilon e^{i \theta}\right)^{\omega+1} f\left(\varepsilon e^{i \theta}\right) \frac{H_{\alpha}^{(1)}\left(\varepsilon e^{i \theta}\right)}{J_{\alpha}\left(\varepsilon e^{i \theta}\right)} d \theta .
\end{aligned}
$$

The asymptotic expansions

$$
\begin{array}{ll}
H_{\alpha}^{(1)}(z) \sim \sqrt{\frac{2}{\pi z}} e^{i(z-\alpha(\pi / 2)-(\pi / 4))}, & |z| \rightarrow \infty,|\arg (z)|<\pi, \\
J_{\alpha}(z) \sim \sqrt{\frac{2}{\pi z}} \cos \left(z-\alpha \frac{\pi}{2}-\frac{\pi}{4}\right), & |z| \rightarrow \infty,|\arg (z)|<\pi,
\end{array}
$$


and the hypothesis (a) show that

$$
\lim _{N \rightarrow \infty} \int_{0}^{M}(x+i N)^{\omega} f(x+i N) \frac{H_{\alpha}^{(1)}(x+i N)}{J_{\alpha}(x+i N)} d x=0 .
$$

The same asymptotic expansions and the hypothesis (b) imply then that

$$
\lim _{M \rightarrow \infty} \int_{0}^{\infty}(M+i y)^{\omega} f(M+i y) \frac{H_{\alpha}^{(1)}(M+i y)}{J_{\alpha}(M+i y)} d y=0 .
$$

Thus, (17) becomes

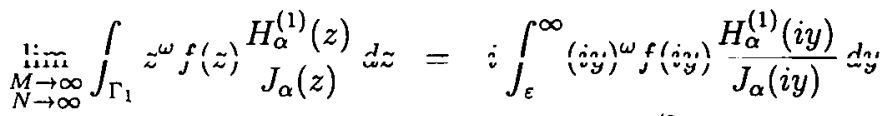

$$
\begin{aligned}
& -i \int_{0}^{\pi / 2}\left(\varepsilon e^{i \theta}\right)^{\omega+1} f\left(\varepsilon e^{i \theta}\right) \frac{H_{\alpha}^{(1)}\left(\varepsilon e^{i \theta}\right)}{J_{\alpha}\left(\varepsilon e^{i \theta}\right)} d \theta .
\end{aligned}
$$

In a similar manner, using the asymptotic expansion

$$
H_{\alpha}^{(2)}(z) \sim \sqrt{\frac{2}{\pi z}} e^{-i(z-\alpha(\pi / 2)-(\pi / 4))}, \quad|z| \rightarrow \infty,|\arg (z)|<\pi,
$$

instead of (18), we obtain

$$
\begin{aligned}
\lim _{\substack{M \rightarrow \infty \\
N \rightarrow \infty}} \int_{\Gamma_{2}} z^{\omega} f(z) \frac{H_{\alpha}^{(2)}(z)}{J_{\alpha}(z)} d z=-i \int_{-\infty}^{-\varepsilon}(i y)^{\omega} f(i y) \frac{H_{\alpha}^{(2)}(i y)}{J_{\alpha}(i y)} d y \\
-i \int_{-\pi / 2}^{0}\left(\varepsilon e^{i \theta}\right)^{\omega+1} f\left(\varepsilon e^{i \theta}\right) \frac{H_{\alpha}^{(2)}\left(\varepsilon e^{i \theta}\right)}{J_{\alpha}\left(\varepsilon e^{i \theta}\right)} d \theta .
\end{aligned}
$$

We infer from (16) that

$$
\begin{aligned}
2 \sum_{k=1}^{\infty} \frac{j_{k}^{\omega-1} f\left(j_{k}\right)}{\left(J_{\alpha}^{\prime}\left(j_{k}\right)\right)^{2}}= & \int_{0}^{\infty} x^{\omega} f(x) d x+\lim _{\varepsilon \rightarrow 0}\left\{-\frac{i}{2} \int_{\varepsilon}^{\infty}(i y)^{\omega} f(i y) \frac{H_{\alpha}^{(1)}(i y)}{J_{\alpha}(i y)} d y\right. \\
& +\frac{i}{2} \int_{-\infty}^{-\varepsilon}(i y)^{\omega} f(i y) \frac{H_{\alpha}^{(2)}(i y)}{J_{\alpha}(i y)} d y \\
& -\frac{i}{2} \int_{0}^{\pi / 2}\left(\varepsilon e^{i \theta}\right)^{\omega+1} f\left(\varepsilon e^{i \theta}\right) \frac{H_{\alpha}^{(1)}\left(\varepsilon e^{i \theta}\right)}{J_{\alpha}\left(\varepsilon e^{i \theta)}\right.} d \theta \\
& \left.+\frac{i}{2} \int_{-\frac{\pi}{2}}^{0}\left(\varepsilon e^{i \theta}\right)^{\omega+1} f\left(\varepsilon e^{i \theta}\right) \frac{H_{\alpha}^{(2)}\left(\varepsilon e^{i \theta}\right)}{J_{\alpha}\left(\varepsilon e^{i \theta}\right)} d \theta\right\}
\end{aligned}
$$

Lsing now the asymptotic expansion

$$
J_{\alpha}(z) \sim \frac{z^{\alpha}}{2^{\alpha} \Gamma(\alpha+1)}, \quad z \rightarrow 0
$$


we obtain

$$
\begin{aligned}
& \lim _{\varepsilon \rightarrow 0} \frac{i}{2} \int_{0}^{\pi / 2}\left(\varepsilon e^{i \theta}\right)^{\omega+1} f\left(\varepsilon e^{i \theta}\right) \frac{H_{\alpha}^{(1)}\left(\varepsilon e^{i \theta}\right)}{J_{\alpha}\left(\varepsilon e^{i \theta}\right)} d \theta \\
&= \begin{cases}2^{2 \alpha-2} \Gamma(\alpha+1) \Gamma(\alpha) f(0) & \text { if } \alpha>0 \\
-\frac{\pi e^{-\alpha \pi i} f(0)}{4 \sin (\alpha \pi)} & \text { if } \alpha<0,\end{cases}
\end{aligned}
$$

and

$$
\begin{aligned}
\lim _{\varepsilon \rightarrow 0} \frac{i}{2} \int_{-\pi / 2}^{0}\left(\varepsilon e^{i \theta}\right)^{\omega+1} f\left(\varepsilon e^{i \theta}\right) \frac{H_{\alpha}^{(2)}\left(\varepsilon e^{i \theta}\right)}{J_{\alpha}\left(\varepsilon e^{i \theta}\right)} d \theta \\
\quad= \begin{cases}-2^{2 \alpha-2} \Gamma(\alpha+1) \Gamma(\alpha) f(0) & \text { if } \alpha>0 \\
\frac{\pi e^{\alpha \pi i} f(0)}{4 \sin (\alpha \pi)} & \text { if } \alpha<0\end{cases}
\end{aligned}
$$

Substituting in (25) we get, for $\alpha>0$,

$$
\begin{aligned}
& 2 \sum_{k=1}^{\infty} \frac{j_{k}^{2 \alpha-2} f\left(j_{k}\right)}{\left(J_{\alpha}^{\prime}\left(j_{k}\right)\right)^{2}}=\int_{0}^{\infty} x^{2 \alpha-1} f(x) d x-2^{2 \alpha-1} \Gamma(\alpha+1) \Gamma(\alpha) f(0) \\
& \quad-\frac{1}{2} \int_{0}^{\infty} y^{2 \alpha-1}\left(e^{\alpha \pi i} f(i y) \frac{H_{\alpha}^{(1)}(i y)}{J_{\alpha}(i y)}+e^{-\alpha \pi i} f(-i y) \frac{H_{\alpha}^{(2)}(-i y)}{J_{\alpha}(-i y)}\right) d y
\end{aligned}
$$

from which Theorem 1 follows since $J_{\alpha}\left(e^{-\pi i} z\right)=e^{-\alpha \pi i} J_{\alpha}(z)$ for $\operatorname{Im}(z)>0$.

Theorem 2 is obtained similarly. In that case $(\alpha<0)$ we have necessarily $f(0)=0$.

\section{EXAMPLES AND REMARKS}

4.1. A GENERALISED RIEMANn $\zeta$ FUnCTION. An interesting application of Theorem 1 arises if we apply it, in appropriate circumstances, to a function of the form $f(z)=$ $1 /(z+\varepsilon)^{\nu}, \varepsilon>0, \nu:=s+2 \alpha-1$. We obtain the relation

$$
2 \sum_{k=1}^{\infty} \frac{j_{k}^{2 \alpha-2}}{\left(J_{\alpha}^{\prime}\left(j_{k}\right)\right)^{2}\left(j_{k}+\varepsilon\right)^{\nu}}=\frac{\Gamma(2 \alpha) \Gamma(s-1)}{\Gamma(s+2 \alpha-1) \varepsilon^{s-1}}-2^{2 \alpha-1} \frac{\Gamma(\alpha+1) \Gamma(\alpha)}{\varepsilon^{s+2 \alpha-1}}
$$

$$
+\frac{e^{-\alpha \pi i}}{2 \sin (\alpha \pi) \varepsilon^{s+2 \alpha-1}} \int_{0}^{\infty} \frac{(i \varepsilon y)^{2 \alpha}}{i y} \frac{\left(J_{\alpha}(i \varepsilon y)-e^{\alpha \pi i} J_{-\alpha}(i \varepsilon y)\right)}{J_{\alpha}(i \varepsilon y)}\left(\frac{1}{(1+i y)^{\nu}}-\frac{1}{(1-i y)^{\nu}}\right) d y
$$

where $\alpha>0$ and $\operatorname{Re}(s)>1$. In the left hand side of $(30)$, we can easily let $\varepsilon \rightarrow 0$. We are led to consider the function $\zeta_{\alpha}$ defined (for all $\alpha$ ) by

$$
\zeta_{\alpha}(s)=2 \pi^{s-1} \sum_{k=1}^{\infty} \frac{1}{j_{k}^{s+1}\left(J_{\alpha}^{\prime}\left(j_{k}\right)\right)^{2}}, \quad \operatorname{Re}(s)>1 .
$$


We readily see that $\zeta_{1 / 2}(s)=\zeta(s)$ and $\zeta_{-1 / 2}(s)=\left(2^{s}-1\right) \zeta(s)$. Also,

$$
\zeta_{3 / 2}(s)=\pi^{s} \sum_{k=1}^{\infty} \frac{\left(1+j_{k}^{2}\right)}{j_{k}^{s+2}}
$$

where $\tan \left(j_{k}\right)=j_{k}$. If we were able to represent suitably a corresponding $\Gamma_{\alpha}$ function then $\zeta_{\alpha}$ would satisfy a practical functional equation analogous to [1, p.259]

$$
\zeta(s)=2(2 \pi)^{s-1} \Gamma(1-s) \sin \left(\frac{\pi s}{2}\right) \zeta(1-s) .
$$

More precisely, we have

$$
\sin \left(\pi \alpha-\frac{\pi s}{2}\right) \zeta_{\alpha}(s)=-i e^{\alpha \pi i} 2^{\alpha-(1 / 2)}(2 \pi)^{s-1} \sin (\pi s) \Gamma_{\alpha}(1-s) \zeta_{\alpha}(1-s),
$$

where $\Gamma_{\alpha}$ is an extension of the classical $\Gamma$ function. The relation $\Gamma(s) \Gamma(1-s)=$ $(\pi / \sin (\pi s))$ becomes the particular case $\alpha=1 / 2$ of

$$
\Gamma_{\alpha}(s) \Gamma_{\alpha}(1-s)=\frac{\pi e^{-2 \alpha \pi i}(\sin (2 \pi \alpha)-\sin (\pi s))}{2^{2 \alpha-1} \sin ^{2}(\pi s)},
$$

which follows from (33).

We don't give details concerning the function $\zeta_{\alpha}$ because there is a difficulty in representing $\Gamma_{\alpha}$ in explicit form. We have only the representation

$$
\Gamma_{\alpha}(s)=\frac{-1}{2^{\alpha+(1 / 2)} \zeta_{\alpha}(s)} \int_{0}^{\infty} r^{s-1} \frac{H_{\alpha}^{(1)}\left(\frac{i r}{2}\right)}{J_{\alpha}\left(\frac{i r}{2}\right)} d r,
$$

with $\operatorname{Re}(s)>2 \alpha$ if $\alpha>0$ and $\operatorname{Re}(s)>0$ if $\alpha<0$. We have $\Gamma_{1 / 2}(s)=\Gamma(s)$ and $\Gamma_{-(1 / 2)}(s)=2\left(\left(2^{1-s}-1\right) /\left(2^{s}-1\right)\right) \Gamma(s)$.

The analytic continuation form of $(31)$ is

$$
\zeta_{\alpha}(s)=\frac{i \pi^{s-1}}{4 \sin (\alpha \pi) \sin ((2 \alpha-s) \pi / 2)} \int_{C} \frac{1}{z^{s}} \frac{\left(J_{\alpha}(-i z)-e^{\alpha \pi i} J_{-\alpha}(-i z)\right)}{J_{\alpha}(-i z)} d z,
$$

where $C$ is a curve starting at $-\infty$ on the negative real axis, surrounding the origin in such a way that the numbers $i j_{k}, k= \pm 1, \pm 2, \ldots$, are excluded from its interior, and returning at $-\infty$ along the negative real axis. The zeros of $\zeta_{\alpha}$ remain to be studied.

4.2. Let $f(z)=(z+\varepsilon)^{\nu} e^{-\delta z}, \varepsilon>0$, with $\operatorname{Re}(\nu)>0, \operatorname{Re}(\delta)>0$ and $|\operatorname{Im}(\delta)|<2$. By letting $\varepsilon \rightarrow 0$ we obtain, from Theorem 1 ,

$$
\begin{aligned}
2 \sum_{k=1}^{\infty} \frac{j_{k}^{2 \alpha+\nu-2} e^{-\delta j_{k}}}{\left(J_{\alpha}^{\prime}\left(j_{k}\right)\right)^{2}}=\frac{\Gamma(2 \alpha+\nu)}{\delta^{2 \alpha+\nu}} & \\
& -\frac{1}{\sin (\alpha \pi)} \int_{0}^{\infty} y^{2 \alpha+\nu-1} \frac{\left(J_{\alpha}(i y)-e^{\alpha \pi i} J_{-\alpha}(i y)\right)}{J_{\alpha}(i y)} \sin \left(\delta y-\frac{\nu \pi}{2}\right) d y .
\end{aligned}
$$


The same example, with $\nu=0$, gives

$$
\begin{aligned}
2 \sum_{k=1}^{\infty} \frac{j_{k}^{2 \alpha-2} e^{-\delta j_{k}}}{\left(J_{\alpha}^{\prime}\left(j_{k}\right)\right)^{2}}= & \frac{\Gamma(2 \alpha)}{\delta^{2 \alpha}}-2^{2 \alpha-1} \Gamma(\alpha+1) \Gamma(\alpha) \\
& -\frac{1}{\sin (\alpha \pi)} \int_{0}^{\infty} y^{2 \alpha-1} \frac{\left(J_{\alpha}(i y)-e^{\alpha \pi i} J_{-\alpha}(i y)\right)}{J_{\alpha}(i y)} \sin (\delta y) d y
\end{aligned}
$$

4.3. As a numerical example related to (2), we take $f(z)=1 /(1+z)^{2}$. The neat result is

$$
\int_{0}^{\infty} \frac{y d y}{\left(1+y^{2}\right)^{2}\left(e^{2 \pi y}+1\right)}=\frac{5}{4}-\frac{\pi^{2}}{8} .
$$

4.4. EXACT QUADRATURE FORMULA. If $f(z)$ is even then, according to our assumptions, it is necessarily analytic in the whole complex plane. In that case, the last integral appearing in (8) is zero, so that

$$
\int_{0}^{\infty} x^{2 \alpha-1} f(x) d x=2 \sum_{k=1}^{\infty} \frac{j_{k}^{2 \alpha-2} f\left(j_{k}\right)}{\left(J_{\alpha}^{\prime}\left(j_{k}\right)\right)^{2}}+2^{2 \alpha-1} \Gamma(\alpha+1) \Gamma(\alpha) f(0),
$$

which is a consequence of $[3$, Theorem 1$]$

$$
\int_{0}^{\infty} x^{2 \alpha+1}(f(x)+f(-x)) d x=\frac{2}{\tau^{2 \alpha+2}} \sum_{k=1}^{\infty} \frac{j_{k}^{2 \alpha}}{\left(J_{\alpha}^{\prime}\left(j_{k}\right)\right)^{2}}\left(f\left(\frac{j_{k}}{\tau}\right)+f\left(-\frac{j_{k}}{\tau}\right)\right)
$$

with $\tau=1$. In fact, (40) follows from (41) where $f(x)$ is replaced by $\left(1 / x^{2}\right)\left(f(x)-\left(2^{\alpha} \Gamma(\alpha+1)\left(J_{\alpha}(x) / x^{\alpha}\right)\right)^{2} f(0)\right)$. Note that $(41)$ is valid for $\operatorname{Re}(\alpha)>-1$. See also [2, Theorem 2] for a more general result of the form (40).

\section{REFERENCES}

[1] T.M. Apostol, Introduction to analytic number theory (Springer-Verlag, Berlin, Heidelberg, New York, 1976).

[2] R. Ben Ghanem and C. Frappier, 'Explicit quadrature formulae for entire functions of exponential type', J. Approx. Theory 92 (1998), 267-279.

[3] C. Frappier and P. Olivier, 'A quadrature formula involving zeros of Bessel functions', Math. Comp. 60 (1993), 303-316.

[4] P. Henrici, Applied and computational complex analysis Vol. 1 and 2 (John Wiley and Sons, New York, 1988 and 1991).

[5] G.N. Watson, A treatise on the theory of Bessel functions, (2nd edition) (Cambridge University Press, Cambridge, 1952).

Département de Mathématiques et de génie industriel

École Polytechnique

C.P. 6079 Succ. Centre-Ville

Montréal (P.Q.)

Canada H3C 3A7

e-mail: clement.frappier@courrier.polymtl.ca 